\title{
ON A PROBLEM OF P. A. SMITH'
}

BY J. C. SU

Communicated by Deane Montgomery, March 31, 1961

1. Introduction. Throughout this note, $Z_{2}$ denotes the group of integers $\bmod 2$ and cohomology means the Alexander-WallaceSpanier cohomology with coefficients in $Z_{2}$. By a cohomology projective $n$-space we mean a compact Hausdorff space $Y$ whose cohomology ring $H^{*}(Y)$ is isomorphic to that of the real projective $n$-space. In [2], Smith proved that if $Z_{2}$ acts effectively on the real projective $n$-space such that the fixed point set $F\left(Z_{2}\right)$ is nonempty, then $F\left(Z_{2}\right)$ has exactly two components $A_{1}$ and $A_{2}$, where $A_{i}$ is a cohomology projective $n_{i}$-space $(i=1,2)$ and $n_{1}+n_{2}=n-1$. Smith then asked whether the result is true if the real projective $n$-space is replaced by a cohomology projective $n$-space. The purpose of this note is to give a positive answer to the question.

We wish to point out that the inclusion of ring structure in the definition of a cohomology projective $n$-space is indispensable as we may see from the following example. Let $Y$ be the one-point union of a 1 -sphere $S^{1}$ and a 2 -sphere $S^{2}$. Clearly $H^{*}(Y)$ as a group is the same as the cohomology group of a projective plane. Let $T$ be a generator of $Z_{2}$ and define the action of $T$ on $Y$ such that on $S^{i}$ it is the reflexion with respect to the diameter passing through the point of contact. Then the fixed point set consists of three isolated points.

2. A construction. The proof of Smith's theorem in [2] has used the fact that a projective $n$-space admits an $n$-sphere as its twofolded covering space. It is therefore quite natural to expect that a cohomology projective $n$-space $Y$ admits a cohomology $n$-sphere as its two-folded covering space. In the following we give a construction of such a cohomology $n$-sphere which is very similar to the construction of a covering space of a pathwise connected, locally pathwise connected, and locally pathwise simply connected space, with the dual of $H^{1}(Y)$ playing the role of fundamental group.

Let $Y$ be a connected compact Hausdorff space and let $\alpha \in H^{1}(Y)$ be a nonzero element. Let $f: Y^{2} \rightarrow Z_{2}$ be a 1-cocycle representing $\alpha$; then there exists an open covering $V$ of $Y$ such that

$$
f\left(y_{0}, y_{2}\right)=f\left(y_{0}, y_{1}\right)+f\left(y_{1}, y_{2}\right) \quad \text { whenever } \quad y_{0}, y_{1}, y_{2} \in V \in v \text {. }
$$

1 The research of this paper is supported by U. S. Army Research Office under Contract No. DA 36-034-ORD-2970. The author also wishes to express his debt to Professor C. T. Yang for his helpful suggestions. 
Fix a point $b \in Y$. By a $v$-chain with base point $b$ we mean a finite sequence $\left(y_{i}\right)_{i=0}^{n}$ of points of $Y$ such that $y_{0}=b$ and $\left\{y_{i-1}, y_{i}\right\}$ is contained in some $V \in V$ for all $i=1,2, \cdots, n$, the set of all $v$-chains with base point $b$ is denoted by $x$. Two $\mathcal{V}$-chains $\left(y_{i}\right)_{i=0}^{n}$ and $\left(y_{j}^{\prime}\right)_{j=0}^{m}$ are said to be equivalent if

$$
\text { (ii) } \sum_{i=1}^{n} f\left(y_{i-1}, y_{i}\right)=\sum_{j=1}^{m} f\left(y_{j-1}^{\prime}, y_{j}^{\prime}\right) \text {. }
$$

The quotient set of $X$ under this equivalence relation is denoted by $X$ and the equivalence class of $\left(y_{i}\right)_{i=0}^{n}$ is denoted by $\left[y_{i}\right]_{i=0}^{n}$.

Now we topologize $X$ as follows. Let $x=\left[y_{i}\right]_{i=0}^{n} \in X$ and $\beta\left(y_{n}\right)$ be a base of neighborhood of $y_{n}$ such that every $B\left(y_{n}\right) \in B\left(y_{n}\right)$ is contained in some $V \in \mathcal{V}$. To each $B\left(y_{n}\right) \in \Re\left(y_{n}\right)$, we define

$$
\begin{aligned}
B^{*}(x)=\left\{\left[y_{j}^{\prime}\right]_{j=0}^{m} \mid y_{m}^{\prime} \in B\left(y_{n}\right), \sum_{i=1}^{n} f\left(y_{i-1}, y_{i}\right)\right. & +f\left(y_{n}, y_{m}^{\prime}\right) \\
& \left.+\sum_{j=1}^{m} f\left(y_{j-1}^{\prime}, y_{j}^{\prime}\right)=0\right\} .
\end{aligned}
$$

It is easily verified that $X$ is made a Hausdorff space with

$$
B(x)=\left\{B^{*}(x) \mid B\left(y_{n}\right) \in B\left(y_{n}\right)\right\}
$$

as a base of neighborhoods of $x$.

Define a map $\pi: X \rightarrow Y$ by $\pi\left(\left[y_{i}\right]_{i=0}^{n}\right)=y_{n}$, it is straightforward to verify that $\pi$ is well-defined and is a local homeomorphism of $X$ onto $Y$.

Obviously, to each $y \in Y, \pi^{-1}(y)$ has at most two points. We now claim that it has exactly two points. To see this, it suffices to consider the case when $y=b$. Since $[b]$ is one point of $\pi^{-1}(b)$, all we have to do is to exhibit a $v$-chain $\left(y_{i}\right)_{i=0}^{n}$ with $y_{0}=y_{n}=b$ and $\sum_{i=1}^{n} f\left(y_{i-1}, y_{i}\right)$ $=1$. Suppose such a chain does not exist, then we can define a 0cochain $g: Y \rightarrow Z_{2}$ by $g(y)=\sum_{i=1}^{n} f\left(y_{i-1}, y_{i}\right)$, where $\left(y_{i}\right)_{i=0}^{n}$ is any $v$ chain with base point $b$ with $y_{n}=y$. Such a chain exists in view of the connectedness of $Y$ and $g$ is clearly well-defined. Now if $\left\{y, y^{\prime}\right\} \in V$ $\in \mathcal{V}$, we have

$$
g\left(y^{\prime}\right)-g(y)=\sum_{i=1}^{n} f\left(y_{i-1}, y_{i}\right)+f\left(y, y^{\prime}\right)-\sum_{i=1}^{n} f\left(y_{i-1}, y_{i}\right)=f\left(y, y^{\prime}\right) .
$$

But this means $f-\delta g$ has empty support, contradicting the assumption that $\alpha \neq 0$.

Now let $T$ be the generator of $Z_{2}$ and define the action of $T$ by exchanging the two points in $\pi^{-1}(y)$ for each $y \in Y$. We clearly obtain 
a free action of $Z_{2}$ on the compact Hausdorff space $X$ with $Y=X / Z_{2}$.

Define a 0 -cochain $h: X \rightarrow Z_{2}$ by

$$
h\left(\left[y_{i}\right]_{i=0}^{n}\right)=\sum_{i=1}^{n} f\left(y_{i-1}, y_{i}\right) .
$$

A similar argument as above shows that $\pi^{*}(\alpha)$ is the cohomology class of $\delta h$.

Suppose that now $Y$ is a cohomology projective $n$-space and that $\alpha$ is the generator of the cohomology ring $H^{*}(Y)$. We claim that $X$ is a cohomology $n$-sphere. As seen in [1], we have the exact SmithGysin sequence

$$
\cdots \rightarrow H^{k}(Y) \stackrel{\pi^{*}}{\rightarrow} H^{k}(X) \stackrel{\tau_{*}^{*}}{\rightarrow} H^{k}(Y) \stackrel{\delta^{*}}{\rightarrow} H^{k+1}(Y) \rightarrow \cdots
$$

Since $\pi^{*}(\alpha)=0$ and $\pi^{*}$ is a ring homomorphism, it follows that $\pi^{*}: H^{k}(Y) \rightarrow H^{k}(X)$ is trivial for all $k>0$. This is enough to conclude that

\section{Main theorem.}

$$
H^{k}(X)=\left\{\begin{array}{lr}
Z_{2}, & k=0, n, \\
0, & \text { otherwise }
\end{array}\right.
$$

THEOREM. If $Z_{2}$ acts effectively on a cohomology projective n-space $Y$ such that the fixed point set $F\left(Z_{2}\right)$ is nonempty, then $F\left(Z_{2}\right)$ has exactly two components $A_{1}$ and $A_{2}$ where each $A_{i}$ is a cohomology projective $n_{i-}$ space $(i=1,2)$ and $n_{1}+n_{2}=n-1$.

Proof. Let $S$ be the generator of $Z_{2}$. In the construction of $X$ given in the last section, we may choose the base point $b$ in $F\left(Z_{2}\right)$ and we may assume that $V$ is $S$-invariant (i.e. $S(V) \in \mathcal{V}$ for all $V \in \mathcal{V}$ ). It follows that $S$ maps $V$-chains with base point $b$ into themselves or $S$ induces a transformation on $x$. Observe that $S$ also induces an automorphism $S^{*}$ on $H^{1}(Y)$; hence we must have $S^{*}(\alpha)=\alpha$. It is easily seen that this fact implies that $S$ maps equivalent $V$-chains into themselves, in other words $S$ induces a transformation $\hat{S}$ on the space $X$ which is clearly compatible with $\pi$ (i.e. $\pi \circ \hat{S}=S \circ \pi$ ). This means we have an action of the group $Z_{2} \times Z_{2}$ on a cohomology $n$-sphere $X$. The rest of the proof is word by word the same as given in [2].

\section{REFERENCES}

1. P. E. Conner and E. E. Floyd, Fixed point free involutions and equivariant maps, Bull. Amer. Math. Soc. vol. 66 (1960) pp. 416-441.

2. P. A. Smith, New results and old problems in finite transformation groups, Bull. Amer. Math. Soc. vol. 66 (1960) pp. 401-415.

University of Pennsylvania 